\title{
Co-Simulation of PCB and Enclosure to Predict Radiated Emission
}

\author{
Yifu Ding, Guangyu Zhang, Xu Zhang, and Zaiyuan Wu \\ China Automotive Technology \& Research Center, Tianjin 300300, China
}

\begin{abstract}
With highly requirement for electromagnetic compatibility (EMC) target, effective controlling of radiated emission of equipment or subsystem is of considerable significance. An emission equivalent model of Printed Circuit Board (PCB) is set up in this paper. Prediction of resonance characteristics, far field radiation of the PCB is carried out and analysed. Then, a 3-D model of a typical equipment enclosure is set up. Resonance characteristics and radiating characteristics of the enclosure is carried out and analysed. A co-simulation method to predict radiated emission is proposed and an example of co-design of PCB and enclosure using HFSS and Siwave verified this method.
\end{abstract}

\section{Introduction}

Electromagnetic compatibility (EMC) problems have been found to be a tricky problem when large numbers of electronic equipment were installed on space-limited electric platform. As an important technical target, EMC has great impact on the efficiency and reliability of electric system. Simulation and prediction of radiated emission effectively in the stage of system design is of great significance to the improvement of EMC performance of system [1].

This paper aims at introducing a method to predict radiated emission of electric equipment in order to optimize the EMC characteristic of electric system. We introduce a co-simulation method to predict the radiated emission of the whole equipment. The co-simulation method is able to control over radiated emission of equipment effectively in the stage of design [2].

\section{Modeling of pcb radiated emission characteristic}

Radiated emission of PCB originated from several types of sources: high frequency signal generated by RC oscillator or crystal oscillator coupling on the traces on PCB and then forming an effective antenna, current and voltage-driven common-mode coupling, ground bounce and etc. Two methods are able to reduce radiated emission of PCB: One method is to control the radiated emission caused by circuit module and traces, the other method is to avoid the resonance between ground and power distribution network (PDN) [3], [4].

Modelling of PCB radiation sources could equivalent the trace or network of highly radiated efficiency to an antenna. Two types of radiation sources could be modelled to equivalent the radiated characteristic of PCB: one is common-mode radiation and the other is differentmode radiation.

\subsection{Different-Mode Radiated Emission}

Different-mode currents flowing through a loop result in different-mode radiated emission. The currents loop could be equalled to magnetic dipole antenna as the different-mode radiated emission model on PCB. An idealized magnetic dipole antenna constitutes of a small currents loop and its radius a meets the inequality: $\mathrm{a}<<\lambda$. We can derive the vector and magnitude of E-field and $\mathrm{H}$-field radiated from the model [5]-[7].

The vector representations of E-field and H-field are:

$$
\begin{gathered}
\mathrm{E}_{\varphi}=-j \frac{\eta_{0} S I k}{4 \pi r}\left(j k+\frac{1}{r}\right) \sin \theta e^{-j k r} \\
H_{\theta}=\frac{I S}{4 \pi r}\left(-k^{2}+\frac{j k}{r}+\frac{1}{r^{2}}\right) \sin \theta e^{-j k r} \\
H_{r}=\frac{I S}{2 \pi r^{2}}\left(j k+\frac{1}{r}\right) \cos \theta e^{-j k r} \\
\left|\mathrm{E}_{\varphi}\right|=-\frac{\eta_{0} S I \pi}{r \lambda^{2}} \sqrt{1+\frac{\lambda^{2}}{4 \pi^{2} r^{2}}} \sin \theta \\
\left|H_{\theta}\right|=\frac{I S \pi}{r \lambda^{2}} \sqrt{1-\frac{\lambda^{2}}{4 \pi^{2} r^{2}}+\frac{\lambda^{4}}{16 \pi^{4} r^{4}}} \sin \theta \\
\left|H_{r}\right|=\frac{I S}{r \lambda^{2}} \sqrt{1+\frac{\lambda^{2}}{4 \pi^{2} r^{2}}} \cos \theta
\end{gathered}
$$

\subsection{Common-mode radiated emission}


Common-mode radiated emission originates from common-mode noise voltage. The noise voltage could be driven in two ways: one is currents-drive voltage noise and the other is voltage-drive voltage noise. Currentsdrive voltage noise caused by voltage drop derives from time-varying different-mode currents flowing through the loop impedance. Voltage-drive voltage noise results from different-mode voltage in circuit network. It is practicable to equal the common-mode radiated emission to an electric dipole antenna. We can derive the vector and magnitude of $\mathrm{E}$-field and $\mathrm{H}$-field radiated from this model.

The vector representations of E-field and $\mathrm{H}$-field are:

$$
\begin{aligned}
\mathrm{E}_{r} & =-j \frac{I d l \cos \theta}{2 \pi \omega \varepsilon_{0} r^{2}}\left(j k+\frac{1}{r}\right) \sin \theta e^{-j k r} \\
E_{\theta} & =-j \frac{I d l \cos \theta}{4 \pi \omega \varepsilon_{0} r^{2}}\left(-k^{2}+\frac{j k}{r}+\frac{1}{r^{2}}\right) e^{-j k r} \\
H_{\varphi} & =\frac{I d l \sin \theta}{4 \pi r}\left(j k+\frac{1}{r}\right) e^{-j k r} \\
\left|\mathrm{E}_{r}\right| & =-\frac{I d l \cos \theta}{2 \pi r^{2}} \sqrt{\frac{\mu}{\varepsilon_{0}}}\left(j+\frac{1}{r k}\right) \\
\left|E_{\theta}\right| & =\frac{I d l \eta_{0} \sin \theta}{2 \lambda r} \sqrt{1-\frac{\lambda^{2}}{4 \pi^{2} r^{2}}+\frac{\lambda^{4}}{16 \pi^{4} r^{4}}} \\
\left|H_{\varphi}\right| & =\frac{I d l \sin \theta}{2 \lambda r} \sqrt{1+\frac{\lambda^{2}}{4 \pi^{2} r^{2}}}
\end{aligned}
$$

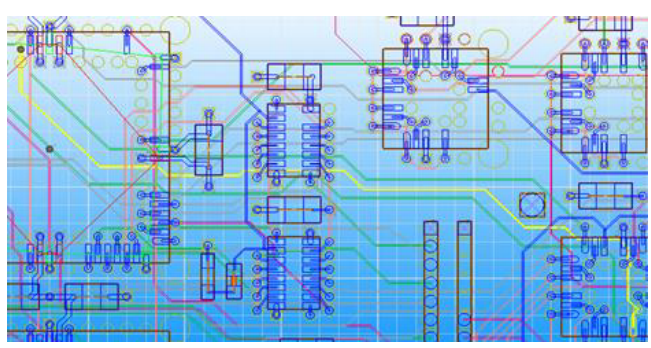

Figure 1. Key Network of a PCB.

\subsection{Modelling of PCB as Radiated Emission Source}

To model PCB radiated emission in a more accurate way, a thorough analysis of the key networks in PCB is necessary. The key networks should include high-speed signal network and power distribution network (PDN)[8]. A key network of an 8-layer PCB analysed in this paper is shown in Fig. 1, the highlighted wire is the high-speed signal network in this board. We put a $1 \mathrm{~V}$ voltage source with $0-1 \mathrm{GHz}$ frequency swept in the network where the coordinate of positive pole is $(X, Y)=(1150,3775)$ and negative pole is $(\mathrm{X}, \mathrm{Y})=(1150,3775)$. The positive is on the surface layer of the $\mathrm{PCB}$ and the negative is on the GND layer of the PCB. The $1 \mathrm{~m}$ far-field radiated emission of PCB is predicted using method of FDTD and the results is shown in Fig. 2.

\section{Co-Simulation of PCB and Enclosure to Control Radiated Emission}

\subsection{Prediction and Optimization to Resonance Character of PCB and Enclosure}

This paper uses an 8-layer PCB shown in Fig. 2 and a typical enclosure as an example to verify the feasibility and effectiveness of the co-simulation method to control radiated emission. We predict the resonant characteristic of the PCB in frequency range of $0.25 \mathrm{GHz}$ to $2 \mathrm{GHz}$ and the calculation results of 5 resonance points shown in Chart 1. Resonance frequency of enclosure depends on its physical shape and the vents, slots, buttons on it. These factors would affect the resonance frequency considerably. In the stage of pre-design, we could simulate and calculate the resonant frequency under the consideration of both resonance and shielding effectiveness in order to reduce radiated emission of the equipment and provide information to the arrangement of PCB and cable inside the enclosure. A typical enclosure is modelled and the dimension and material properties are shown in Table 1. And then we predict the resonant characteristic of the enclosure in frequency range of 0.25 to $2 \mathrm{GHz}$ and the calculation results of 5 resonance points is shown in Chart 2.

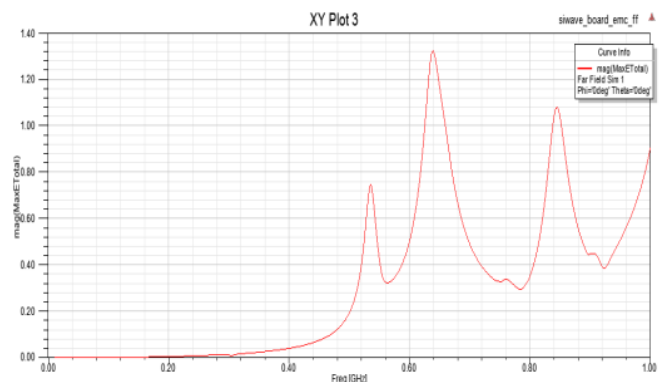

(a)
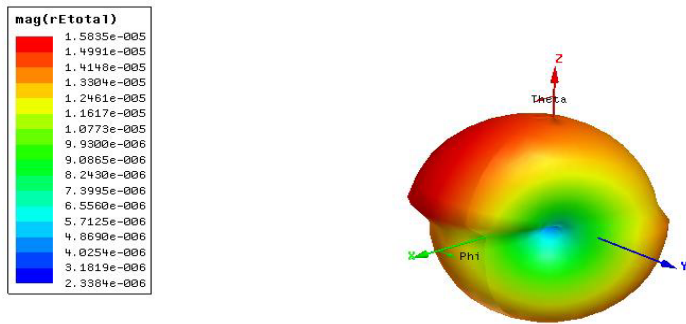

(b)

Figure 2. The $1 \mathrm{~m}$ Radiated Emission of PCB

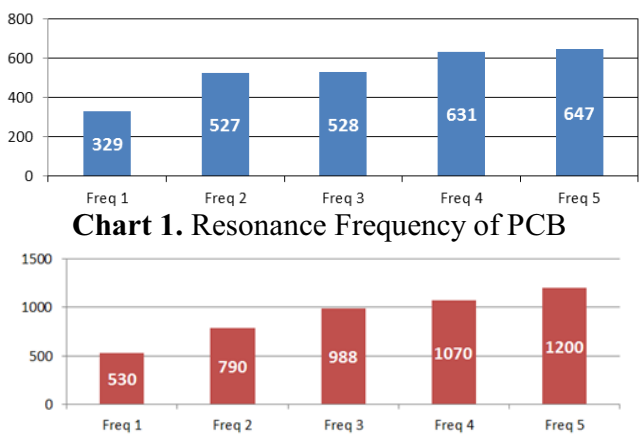

Chart 2. Resonance frequency points of enclosure. 


\section{ICMMR 2016}

Table 1. Dimension and material properties of enclosure

\begin{tabular}{cc}
\hline Dimension & $200 \mathrm{~mm} * 200 \mathrm{~mm} * 100 \mathrm{~mm}$ \\
\hline Thickness & $2 \mathrm{~mm}$ \\
Material & Aluminum \\
Radius of aperture & $10 \mathrm{~mm}$ \\
Position of aperture & $(198,100,60)$ \\
\hline
\end{tabular}

\subsection{Radiated emission of equipment and co- design}

In order to predict the radiated emission of the equipment (PCB inside enclosure), we modelled the PCB as an equivalent antenna (in chapter II) as a radiated source and then the enclosure is modelled as a 2 nd time radiated source. This method avoids the inaccuracy computation of direct division to the enclosure and PCB. Then we modelled an equivalent $\mathrm{PCB}$ as a material of metallic surface and dielectric interlayer inside the enclosure with the dimension of $200 \mathrm{~mm}$ length, $100 \mathrm{~mm}$ width and $10 \mathrm{~mm}$ thickness. The extracted equivalent radiated source is put on the position of $(0,80,0)$. Simulation result of the E-field distribution is shown in Fig 3.

Table 2. Optimized Enclosure Settings

\begin{tabular}{cc}
\hline Vents Distribution & $2 \times 2$ \\
\hline Shape of Vents & Round \\
& \\
Coordinates of Vents & $(198,50,20)(198,50,40)$ \\
& $(198,30,20)(198,30,40)$ \\
Radius of Vents & $2.5 \mathrm{~mm}$ \\
\hline
\end{tabular}

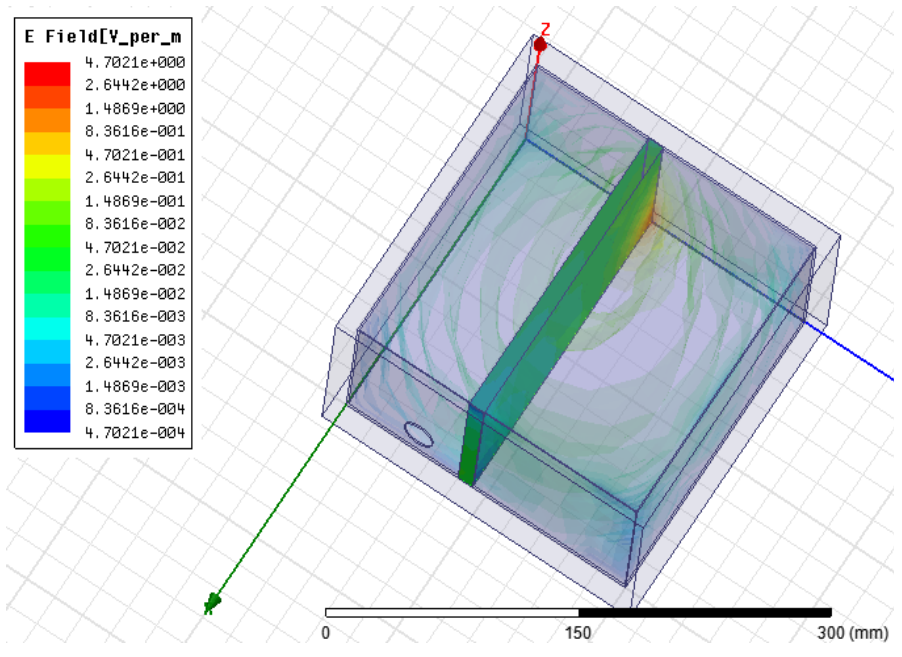

Figure 3. Distribution of E-field of the equipment.

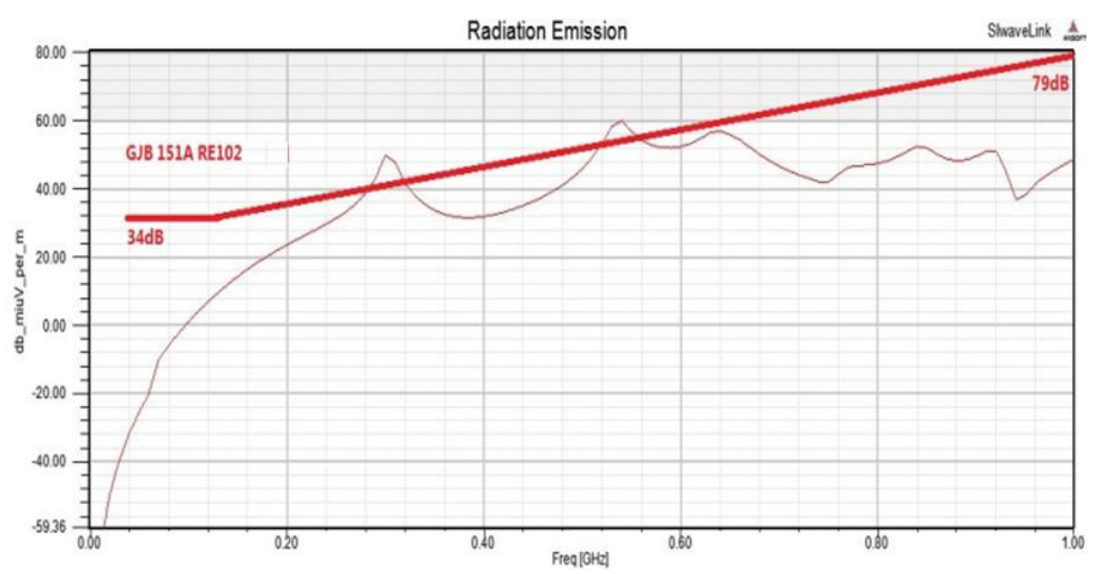

Figure 4. Simulated RE102 test results.

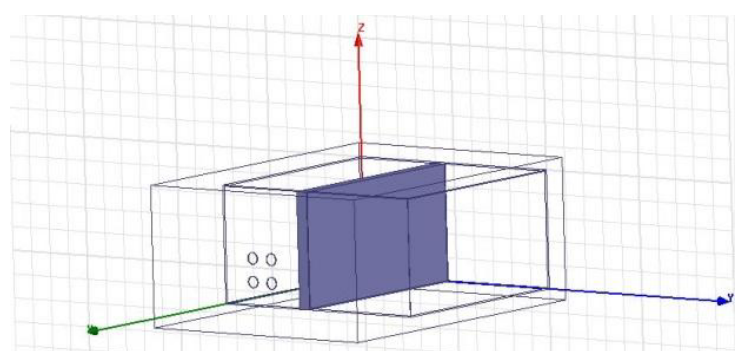

Figure 5. Optimized enclosure. 


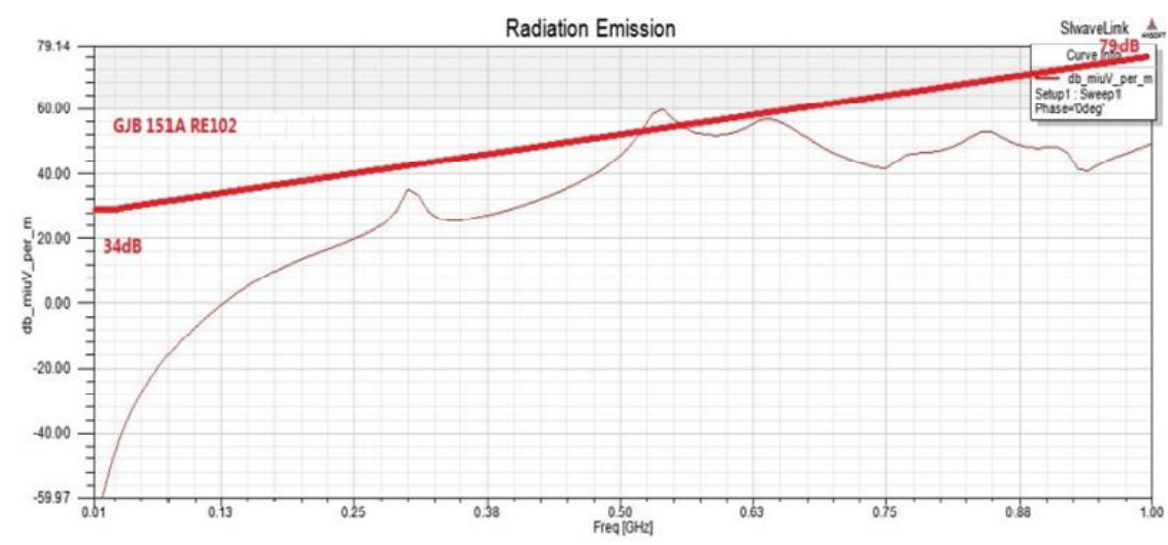

Figure 6. Simulated RE102 test results.

In order to verify the co-simulation method in design of equipment, an optimization to the radiated emission of the equipment by the reference of radiated emission limits of GJB151A is carried out. The simulation result is shown below in Fig. 4.

Obviously, there exists two frequency points of overradiated emission under the RE102 test: a $7 \mathrm{~dB}$ over radiated emission on the frequency of $240 \mathrm{MHz}$ and a $6 \mathrm{~dB}$ over radiated emission on the frequency of $570 \mathrm{MHz}$. The equipment cannot be certified in this level of radiated emission and an optimization is necessary. In order to maintain the same level of heat output performance, the whole area of the vent cannot be changed and according the theory of the effects of vent. The dimension and distribution of vents of the optimized enclosure is shown in Table 2 and the optimized enclosure is shown in Fig. 5.

The radiated emission of the optimized equipment is shown in Fig. 6. Therefore, the optimized equipment is able to be certified in the RE102 test and the radiated emission in the frequency point $340 \mathrm{MHz}$ is $33 \mathrm{~dB} \mu \mathrm{v} / \mathrm{m}$, which has a $15 \mathrm{~dB}$ reduction from the original one.

\section{Summary}

In this paper, we conclude the influence of resonance character to the radiated emission of equipment and modelled the equivalent antenna to PCB radiated emission source. Using the source, as an excitation inside the enclosure, to predict radiated emission of the equipment. Thus, a co-simulation method to predict radiated emission is proposed and an example of codesign of PCB and enclosure using HFSS and Siwave verified this method. The simulation and prediction results were contrasted with radiated emission maximum limits required by GJB151A Standard and verified the correctness and efficiency of the method. This method is able to provide theoretical foundation and design method for the radiated emission control in the stage of predesign of electric equipment and system.

\section{References}

1. Ji ZHANG, Juan SHI, Tengfei Yang. Radiated emission test and its reduction of PCB [J]. Journal of Jiamusi University, 2012, v.30; No.12005:706$709+713$.

2. Yang ZHAO, Wei YAN, Bo ZHAO, Y.C LUO, Shijin LI. Evaluation and Tests of Circuit Radiated EMI [J]. DIANGONG JOURNAL, 2010, v.2510:613.

3. Christian Poschalko, IEEE TRANS ON EMC [J]. Vent Model for the Slot Radiation of an Enclosure Excited by Printed Circuit Board Traces with Different Loads.

4. M. Leone, "Design expressions for the trace-to-edge common-mode inductance of a printed circuit board," IEEE Trans. Electromagnetic. Compatibility. vol. 43, no. 4, pp. 667-671, Nov. 2001.

5. H.-W. Shim and T. H. Hubing, "Model for estimating the radiated emissions from a printed circuit board with attached cables due to voltage driven sources," IEEE Trans. Electromagnetic Compatibility, vol. 47, no. 4, pp. 899-907, Nov. 2005.

6. D. M. Hockanson, J. L. Drewniak, T. H. Hubing, and T. P. Van Doren,"FDTD modeling of common-mode radiation from cables," IEEE Trans on Electromagnetic Compatibility., vol. 38, no. 3, pp. 376-387, Aug. 1996.

7. D. M. Hockanson, X. Ye, J. L. Drewniak, T. H. Hubing, T. P. Van Doren, and R. E. Du Broff, "FDTD and experimental investigation of EMI from stacked-card PCB configurations," IEEE Trans. Electromagnetic Compatibility. vol. 43, no. 1, pp. 110, Feb. 2001

8. J. E. Bracken, S. Poltyanko, S. Raman, and Z. J. Cendes, "Efficient full wave simulation of highspeed printed circuit boards and electronic packages," in Proc. IEEE Antennas Propagation. Soc. Int. Symp. 2004, Jun. 20-25, vol. 3, pp. 3301-3304. 